\section{Neurological deterioration in young adults with phenylketonuria}

SIR,-Since diet treatment began for phenylketonuria (PKU) 30 years ago several caveats have emerged: ( $a$ ) neonatal diagnosis is essential and is the basis of screening programmes, though this time frame for diagnosis is controversial; ${ }^{1}(b)$ excessive dietary restriction of essential aminoacids is harmful to infant growth and development; and (c) "IQ deterioration" has followed diet termination before 10 years of age in some studies. ${ }^{2}$ Dr Thompson and colleagues (Sept 8, p 602) extend the concern over diet termination leading to neurological deterioration in young adults with PKU. These caveats, however, lack scientific support. There are no case-control cohort data, such as meta-analyses, that examine hypotheses affecting PKU health-care attitudes; and ethical concerns prevent scientific cohort studies in infants and children. Nonetheless, this model of a treatable, rare metabolic disorder that is limited in clinical neuroepidemiological information examined by cost-effective analyses does influence national child health policies.

While the neurological symptoms observed by 'Thompson et al could be a sign of central nervous system (CNS) deterioration, independent neurological progression from previous brain insult or the appearance of another concurrent or contiguous disease should be considered. In the absence of prevalence data and case-control unvestigations conflicting viewpoints are not uncommon in PKU management. Specific CNS molecular mechanisms have not been established for this metabolic encephalopathy. There are, however, many excellent scientific observations, such as those on movement disorders, that expand speculation about aetiology. Disordered metabolism of myelin must now be supplemented with the evidence of neurochemical perturbations of neurotransmitters, co-factors, the blood-brain-barrier, and neurocellular development. Histopathological studies on in-utero PKU fetal brain injury reveal dendritic abnormalities with normal myelination. ${ }^{3}$ Brain ontogeny and plasticity in the milieu of excitatory aminoacids suggest a common ground between brain insults such as hypoxia-ischaemia and metabolic disease. ${ }^{4}$

Thompson et al suggest diet termination as the most likely explanation of the neurological symptoms. However, the infant PKU brain, damaged by delayed treatment, may respond differently to a recurrent biochemical insult. 3 of the 7 infants were diagnosed and treated at ages of 15,18 , and 24 months, well beyond the average age of neonatal screening. 2 had infantile spasms, an ominous sign of permanent brain injury from any origin. In our 25-year experience only babies missed by neonatal screening and treated after 6 months of age acquire this disorder. In patients 1 and 3 , with "poor growth", there could be a relation with nutritionally deficient diets. Furthermore, patient 5, with dystonia without a co-factor abnormality, resembles an adult dystonic patient with biopterin deficiency. ${ }^{5}$ Patient 2 , with pyramidal weakness and unknown phenylalanine levels who had been "on diet" for 16 years is clinically similar to the spasticity and parkinsonism described in PKU at different ages. Idiopathic dystonia responds to pterin treatment, as do patients with co-factor deficiency.

Dystonia is a regional neostriatal disorder, often without neuropathological abnormalities such as demyelination but with several monoaminergic abnormalities. Dystonia is not an uncommon idiopathic neurological symptom. Late-onset progressive dystonia after neonatal hypoxic-ischaemic brain damage is well known and could include the brain damage of neonatal metabolic disorders. Late-onset pterin-deficiency dystonia developing in childhood after screening in infancy responds to blopterin treatment but not to diet. ${ }^{6}$ Dystonia and parkinsonism in prenn-deficient variant PKU may appear in infancy. ${ }^{7.8}$

PKU infants and children while under biochemical control may manfest major second disorders such as microcephaly, motoneuron drseases, and ataxia telangiectasia; minor structural abnormalities are also observed in metabolic disorders. The findings in latetreated adults are difficult to differentiate from multiple sclerosis. ${ }^{9}$ Eien phenylalanine in the dietary sweetener aspartame has been implicated in neurological symptoms ${ }^{10}$ in PKU.

Our neurological study consists of a cohort of 309 newborn babies with PKU detected in 3 million births, representing total ascertainment in a population of 10 million people between 1965 and 1990. PKU adults identified before and after this period are under long-term neurological observation; only the 1 infant with variant PKU is dystonic. Caveats about dietary treatment in PKU still await pathophysiological and neuroepidemiological data.

Section of Pediatric Neurology.

Department of Pediatrics and Neurology

University of Michigan Medical School,

Ann Arbor, Michigan 48109, USA

RICHARD J. ALLEN

1. Pietz J, Benninger $\mathrm{Ch}$, Schmidt $\mathrm{H}$, et al. Long-term development of intelligence (IQ) and EEG in 34 children with phenylketonuria treated early. Eur 7 Pediatr 1988; 147: $361-67$.

2. Michals K, Azen C, Acosta P, Koch R, Matalon R. Blood phenylalanine levels and intelligence of 10-year-old chuldren with PKU in the National Collaborative Study. f Am Diet Assoc 1988; 88: 1226-29.

3. Lacev DJ, Terplan $\mathrm{K}$ Abnormal cerebral cortical neurons in a child with maternal PKU syndrome. $\mathcal{I}$ Child Neurol 1987; 2: 201-04.

4. Hattori $H$, Wasterlain CG. Exctatory amino acids in the developing brain: ontogeny, plasticity, and excitotoxicity Pediatr Neurol 1990; 6: 219-28.

5. Tanaka $K$, Yoneda $M$, Nakajma $T$, Miyatake $T$, Oweda $M$. Dihydrobıopterin synthesis defect: an adult with diurnal fluctuation of symptoms. Neurology 1987; 37: 519-22.

6. Fink JK, Barton N, Cohen W, Lovenberg W, Burns RS, Hallett M. Dystonia with marked diumal variation associated with biopterin deficiency Neurology 1988; 38: 707-11.

7. Brismar J, Aqeel A, Gascon G, Ozand P. Malignant hyperphenylalaninemia, CT and MR of the brain. AfNR 1990; 11: 135-38.

8. Allen RJ, Young W, Bonacci I, Persico S, Andruszewski K, Schaefer AM. Neonatal dystonic parkinsonism, a "stiff baby syndrome" in biopterin deficiency with hyperprolactınemı detected by newborn screening for hyperphenylalaninemı. Arm Neurol 1990, 28: 320 (abstr).

9. Villasana D, Butler IJ, Willams JC, Roongta SM, Neurological deterioration in adult phenylketonuria. I Inher Metab D2s 1989; 12: 451-57.

10. Matalon R, Michals K, Sullivan D, Wideroff L, Levy P. Aspartame consumption in normal individuals and carners for phenylketonuria. In: Wurtman RJ, RitterWalker E, eds. Dietary phenylalanine and brain function. Boston: Birkhauser, 1988: 41-52.

\section{Phyllanthus amarus and hepatitis B}

SIR,-Dr Leelarasamee and colleagues (June 30, p 1600) report the failure of Phyllanthus amarus to eradicate hepatitis B surface antigen from symptomless carriers. Our Lancet paper $^{1}$ on the effects of $P$ amarus in chronic carriers of hepatitis B virus (HBV) drew comments in the journal ${ }^{2}$ and via personal communication. The main criticisms of our preliminary study ${ }^{1}$ were that HBV-DNA estimations were not done in the pretreatment and post treatment samples of the cleared and refractory carriers; that HBeAg to anti-HBe seroconversion was not fully evaluated; and that longterm carriers (ie, those who carry $\mathrm{HBsAg}$ for a year or more) were not studied.

With these criticisms in mind we did an open trial in 1989-90 on 28 symptomless chronic HBV carriers known to have carried HBsAg for at least a year and up to 5 years. They were treated with $250 \mathrm{mg}$ capsules of $P$ amamus thrice daily for 3 months. Serum was obtained before treatment, once a month during treatment, and after treatment up to one year of follow-up. Sera were screened for HBV serological markers (HBsAg seroconversion, anti-HBc IgM, $\mathrm{HBe}$ seroconversion) with Organon ELISA kits. HBV-DNA analysis was done by dot-blot hybridisation, ${ }^{3}$ the probe being kindly provided by Dr Stephen Locarnini (Fairfield Hospital, Melbourne, Australia).

Analysis of the data after the treatment period revealed loss of HBsAg in $20 \%$ of carriers, in contrast to $59 \%$ in our earlier study. ${ }^{1}$ All those who have cleared $\mathrm{HBsAg}$ were $\mathrm{HBeAg}$ negative carriers. Of the $4 \mathrm{HBsAg}$ cleared cases, 3 seroconverted to anti-HBs. None seroconverted from $\mathrm{HBeAg}$ positivity to anti-HBe positivity (table). On analysis of HBV-DNA, 15 showed pretreatment positivity (8/11 HBeAg positive, $7 / 9 \mathrm{HBeAg}$ negative). After treatment with $P$ amarus in 2 out of $8 \mathrm{HBeAg}$ positive cases and 4 out of 7 in the $\mathrm{HBeAg}$ negative group, HBV-DNA was not detectable in post treatment sera. $\mathrm{HBeAg}$ positive carriers seem to go through a sequential pattern of $\mathrm{HBe}$ seroconversion followed by a reduction in HBV-DNA levels during treatment before HBV-DNA becomes undetectable. Long-term $P$ amarus treatment of $\mathrm{HBeAg}$ positive carriers for at least 6 months at a dose of $500 \mathrm{mg}$ thrice daily might yield better results.

Pamarus is being studied in a multicentre trial in HBV carriers in New Zealand, Vanavatu, Australia, Egypt, Singapore, China, the 